\title{
Reflux control of a laboratory distillation column via MPC-based reference governor
}

\author{
Martin Klaučo, Richard Valo, Ján Drgoňa \\ Slovak University of Technology in Bratislava, \\ Radlinského 9, SK-812 37 Bratislava, Slovak Republic \\ martin.klauco@stuba.sk,richard.valo@stuba.sk,jan.drgona@stuba.sk
}

\begin{abstract}
An optimization-based control strategy is proposed to improve control performance of a primary PI controller. The strategy, referred to as a MPC-based reference governor, optimizes the performance of a primary PI controller by supplying optimal setpoints to the primary controller. This primary PI controller is responsible for reflux ratio manipulation in a distillation column, to control the temperature of the column head. This paper shows theoretical and experimental results obtained on the laboratory-scale.
\end{abstract}

\section{Introduction}

In the control of a distillation process, control engineers focus on several key aspects. First and foremost, the control strategy must ensure the quality of the product, i.e., distillate. Secondly, since the distillation process is an energy demanding process, the control strategy should be designed to decrease the energy demand. Moreover, to ensure smooth and safe operation, technological and safety limitations have to be obeyed.

The distillation process, however, is often controlled using simple PI controllers (Mihal' et al., 2010; Skogestad, 2007), which have one advantage. Their structure is very simple; hence they are easy to implement and tune. On the other hand, it is near impossible to incorporate safety and technological constraints into the design of PI controllers.

This paper discusses a novel approach based on optimization. Specifically, an extended Model Predictive Control (MPC) strategy is discussed and used as a supervisory controller to the primary PI controller. A similar control strategy was used in early 70 's when the MPC-based strategy was first introduced in the control of distillation processes and oil refineries (Cutler and Ramaker, 1979). Such a concept is often referred to as a reference governor control (Gilbert and Kolmanovsky, 2002). The main advantage of using an MPC-based control strategy is systematic constraints handling. Moreover, several quality and economic criteria can be included in the MPC design, e.g., minimization of used energy as proposed by Martin et al. (2013). Pure MPC approaches have been discussed in relation to the control of distillation columns by many researchers like Volk et al. (2005), Ahmad and Wahib (2007) or by Drgoňa et al. (2016). However, incorporating optimal control strategies like MPC is a costly process. Namely, it requires changing the current control infrastructure. In our work, we propose to keep the current control scheme and to add the optimization layer on top of the current control scheme. Such an upgrade does not require changing the technological layout. The main motivation for this paper is to demonstrate that the MPC-based layer can provide such a setpoint for the inner PI controller that constraints on control action are handled in optimal fashion, even if the PI controller is not well-tuned.

The Theoretical Section is devoted to the description of the laboratory distillation column and the design of the MPC-based reference governor (MPC-RG). In the Experimental Section, control performance of the MPC-based reference governor is compared with the traditional PI controller. In the last section, conclusions are drawn.

\section{Theoretical}

The objective of this paper is to apply and verify a novel control approach involving an MPC-based reference governor as a supervisor controller to a simple PI controller. A laboratory-scale distillation column, where the separation of a methanol-water mixture takes place, was chosen as the controlled process. The control setup involves a single-input single-output system, where input $u$ to the system is the reflux, which directly affects the temperature of the column head considered as a measured variable $y$. Note that the temperature of the column head can be directly recalculated to the product concentration via phase equilibrium diagrams.

The scheme of the continuous laboratory distillation column is presented in Fig. 1. The laboratory distillation column UOP3CC (Armfield, 2010) consists of a reboiler with the maximum heating power of $2.5 \mathrm{~kW}$ and the capacity of $20 \mathrm{dm}^{3}$. In the reboiler, a solution of methanol and water is heated up to the boiling point. From the reboiler, vapor enriched with methanol molecules rises through nine trays to the overhead water-cooled condenser. Condensed 
product is directed through the reflux valve either to the distillate accumulator or it is returned back to the column. The feed mixture is pushed to the column via a peristaltic pump on tray No. 5, and is preheated by a separate electrical heater. Each tray is equipped with a thermocouple. During the distillation column operation, the overhead condenser and the electrical preheater were assumed to be controlled by a separate algorithm and not to be part of the discussed research. These algorithms ensure that the feed is preheated to the boiling point of the feed mixture and the product is not over-cooled, so it can be returned back to the column without disrupting the column operation.

Quality of the distillate is controlled primarily by the reflux valve. More specifically, the concentration of the final product is dependent on the temperature of the column head, i.e. tray No. 9. In our control setup, temperature of the column head was the process variable controlled via a PI controller, which is given in the form of a transfer function

$$
U(s)=P\left(1+\frac{I}{s}\right),
$$

where the variable $U(s)$ is the control action in the Laplace $s$-domain. Variables $P$ and $I$ are tuning parameters of the PI controller. Variable $u$ represents the manipulated variable, which is the reflux ratio

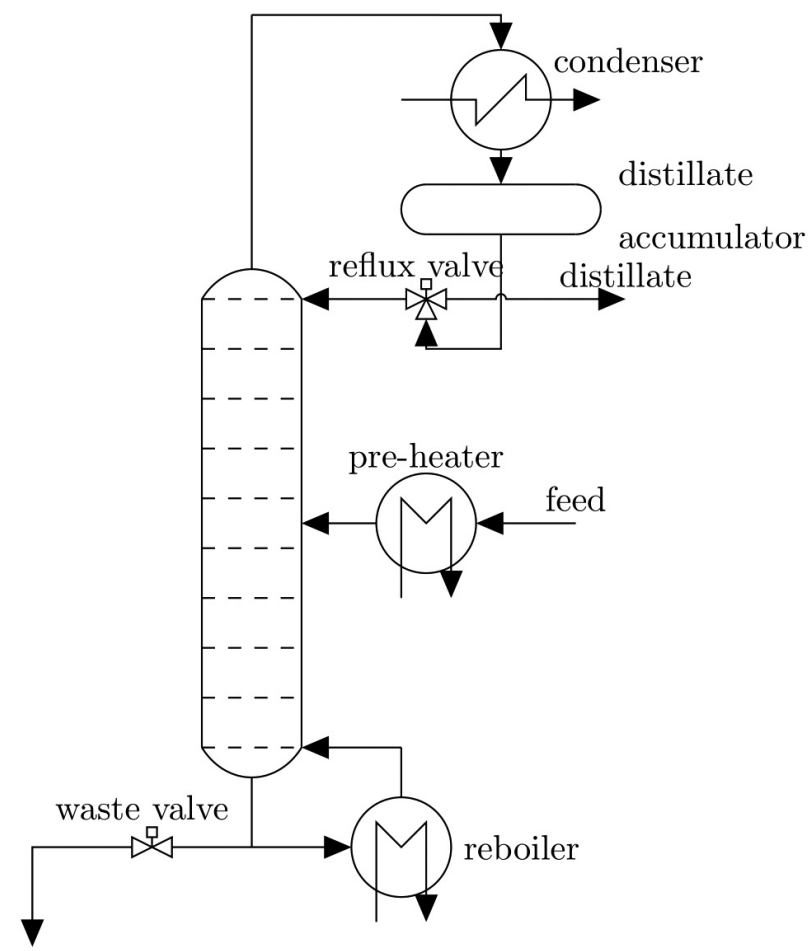

Fig. 1. Scheme of the laboratory distillation column. Opening of the reflux valve is the manipulated variable $u$, while temperature of the column head is the process variable $y$.
$R$. The reflux ratio is constrained in the interval $R \in\left[\begin{array}{ll}0 & 100\end{array}\right] \%$. If the reflux ratio is set to $R=0 \%$, full stream of the condensed product is returned back to the column, while setting $R=100 \%$ results in directing the entire stream to the product accumulator. Naturally, such constraints on the control action can be incorporated in the PI control design using the antiwind-up scheme of Åström and Hägglund (2006); however, performance of such control strategy is not optimal for constrain handling.

The objective of this paper is to devise an MPCbased reference governor which supplies an optimal setpoint to the PI controller; hence, optimal operation of the distillation column is achieved even if a non-optimal PI controller is used. These optimal setpoints ensure the limitations on the reflux ratio not to be violated. The scheme of the proposed control strategy is depicted in Fig. 2. Optimally shaped reference is denoted by $w, e$ denotes the control error, while $u$ and $y$ represent the actual control action and measurement, respectively.

The MPC-based reference governor is formulated as a constrained finite time optimization control (CFTOC) problem. The formulation resembles standard MPC formulations used to control distillation columns (Muske and Badgwell, 2002).

Specific formulation of the MPC-RG is given as

$$
\begin{gathered}
\min _{w_{0}, \ldots, w_{N-1}} \sum_{k=0}^{N-1}\left(\left\|y_{k}-r\right\|_{Q_{y}}^{2}+\left\|\Delta w_{k}\right\|_{Q_{w}}^{2}+\left\|\Delta u_{k}\right\|_{Q_{u}}^{2}\right) \\
x_{k+1}=A x_{k}+B w_{k}, \quad \forall k \in \mathbb{N}_{0}^{N-1}, \\
u_{k}=C_{u} x_{k}+D_{u} w_{k}, \quad \forall k \in \mathbb{N}_{0}^{N-1}, \\
y_{k}=C_{y} x_{k}+D_{y} w_{k}, \quad \forall k \in \mathbb{N}_{0}^{N-1}, \\
u_{\min } \leq u_{k} \leq u_{\max }, \quad \forall k \in \mathbb{N}_{0}^{N-1}, \\
x_{0}=x(t)
\end{gathered}
$$

where the objective function is given by $(2 \mathrm{a})$ and defines the quality criterion. Expression $\|z\|_{M}^{2}$ repre-

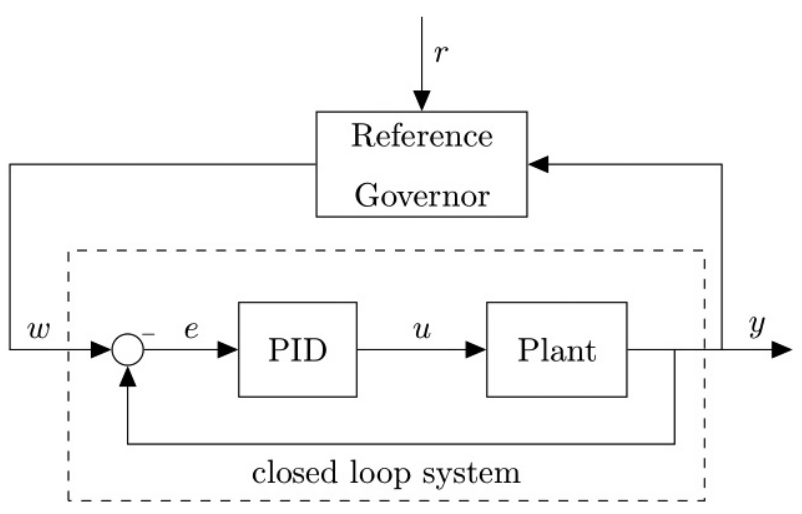

Fig. 2. Scheme of the reference governor setup. 
sents a squared Euclidean norm, i.e. $\|z\|_{M}{ }^{2}=z^{T} M z$. The first term of the cost function penalizes the differences between user-defined reference, $r$, and the predicted measurement, $y$, the second and third terms penalize the fluctuations of the provided setpoint and actual control actions, respectively. Constraints (2b)(2d) represent the model of the closed-loop system. Bounds of the control action, i.e. the reflux, are enforced by (2e). Finally, the optimization problem is initialized by the current state measurement $x(t)$.
The model of the closed-loop system is derived from the identified model of the distillation column and the model of the PI controller. The identified model of the distillation column is taken from our previous work presented in Drgoňa et al. (2015). Details on the closed-loop system modeling derivation can be found in the technical manual by Klaučo (2016). Experimental results obtained via the MPC-RG strategy are presented in the next section.

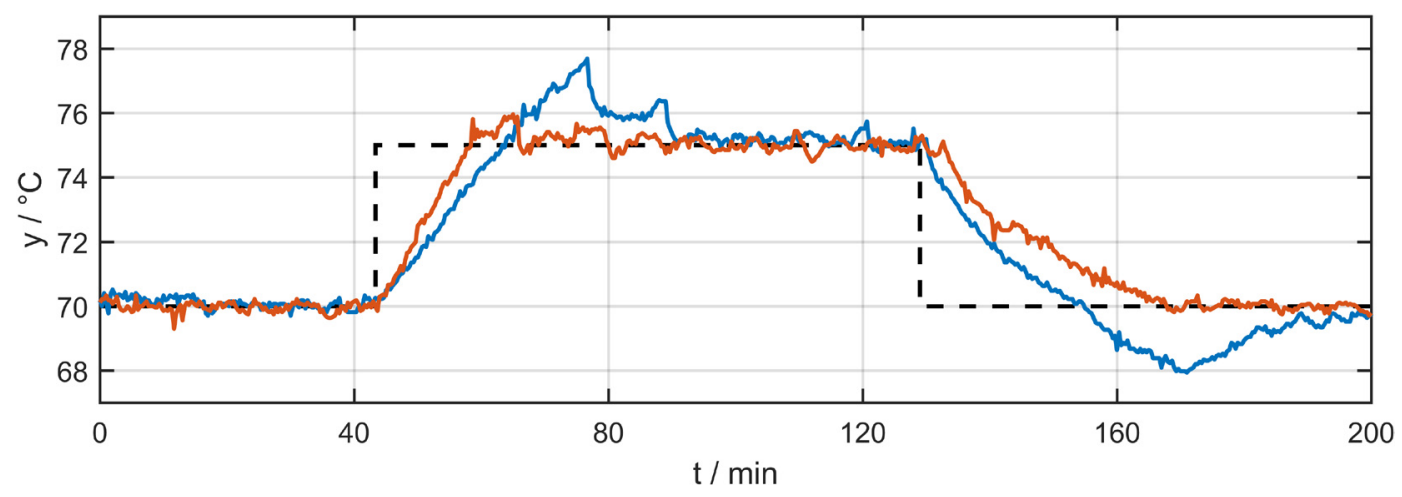

a) Measured temperature of the column head.

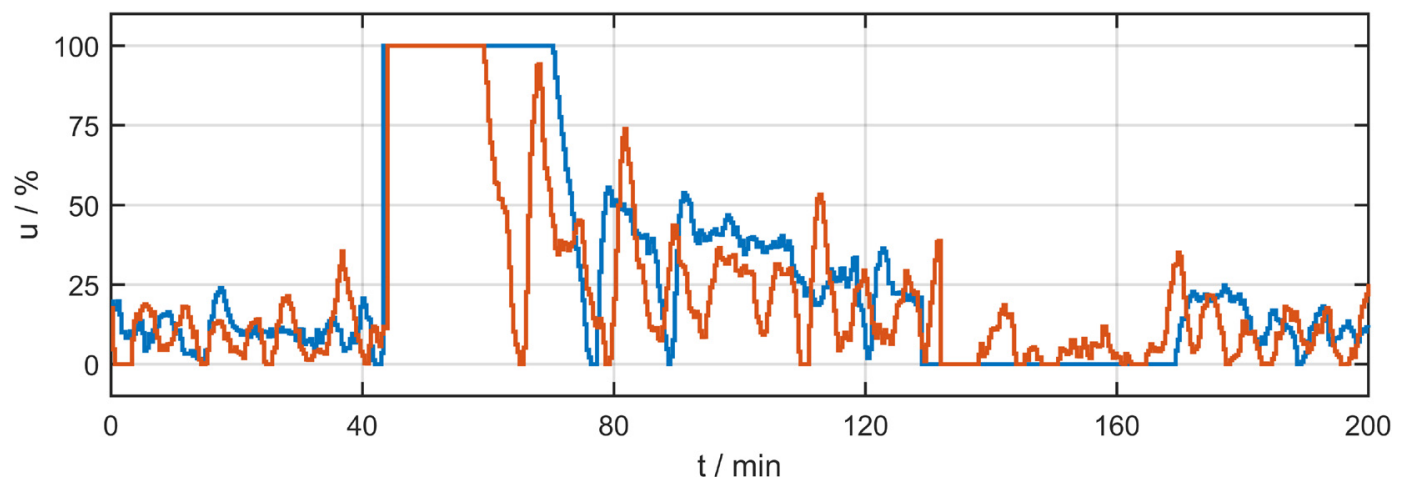

b) Shaped setpoint for the PI controller.

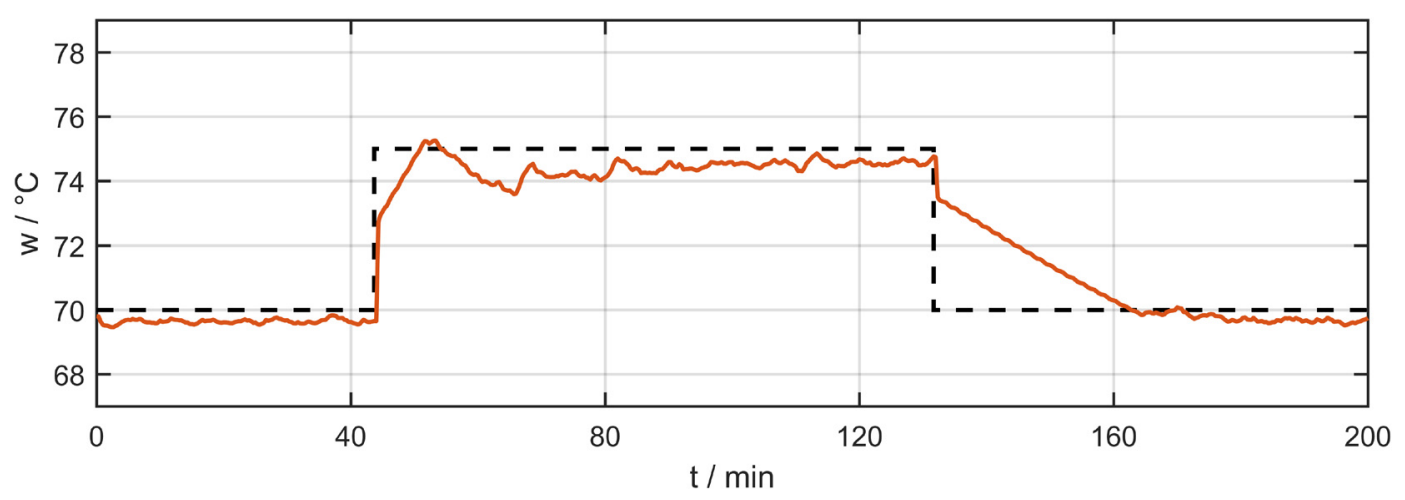

c) Reflux ratio.

Fig. 3. Measurement profiles under the MPC-RG control authority. Dashed line depicts the user defined reference change, while the orange solid line represents measurements of the temperature, shaped setpoint, and the reflux ratio, respectively. Blue solid curve depicts profiles with the PI controller. 


\section{Experimental}

This section covers experiments in a laboratoryscale distillation column. Two types of experimental scenarios were performed. The first one involved the control of the reflux via the novel MPC-based reference governor strategy. The second scenario was limited to the traditional control approach, the PI control. The sampling time in both scenarios was set to $T_{\mathrm{s}}=10 \mathrm{~s}$.

The MPC-based controller was designed based on the model of the closed-loop system involving the identified model of the distillation column (Drgoña et al., 2015) and the model of the PI controller in form (1). Tuning parameters of the PI controller were set to

$$
\mathrm{P}=55, \mathrm{I}=0.011
$$

The prediction horizon of the governor was chosen as $N=30$, while the tuning factors in the objective function were set to

$$
Q_{\mathrm{v}}=50, Q_{\mathrm{v}}=10^{-3}, Q_{\mathrm{u}}=10^{-3}
$$

Finally, technological limitations on the reflux ratio were considered, hence:

$$
\mathrm{u}_{\min }=0, \mathrm{u}_{\max }=100
$$

The control problem formulation of the MPC-based governor has been constructed by using YALMIP Löfberg (2004). The resulting optimization problem was solved by the GUROBI solver at each sampling instant. The performance of both controllers was tested on a scenario, where a step change on the setpoint $r$, i.e. temperature of the column head was changed. First, the experiment with the novel control strategy involving the MPC-based reference governor was tested. Results of this experiment are shown in the Fig. 3. Recall, that the controlled variable $y$ is the temperature of the column head. The profile of the variable is shown in the Fig. 3a, while the optimally shaped setpoint for the inner controller $w$ is depicted in the Fig. 3c. Lastly, the profile of the reflux ratio, which is the manipulated variable, is shown in the Fig. 3b.

In the second experiment the temperature of the column head was controlled solely by the PI controller (1). Time profiles of the measured temperature and reflux ratio are provided alongside the results obtained using MPCRG in Figs. 3a and 3b, respectively.

Extending the primary closed-loop by MPCRG, the temperature overshoot (see the Fig. 3a) in the column head is negligible compared to the case using the PI controller only. Also, counteracting the overshoot by shaping the reference (see Fig. 3b around time $t=50 \mathrm{~min}$ ), the reflux ratio valve changes position from $u=100 \%$ approximately 7 min sooner compared to the scenario involving the PI controller only. This behavior can be seen in the Fig. 3b. Moreover, the IAE criterion defined as

$$
\mathrm{IAE}=\sum_{k=0}^{t_{f}}\left|y_{k}-r_{k}\right|
$$

was considered to compare the performance of the respective controllers. Variable $t_{f}$ represents the entire experiment time window, i.e. $200 \mathrm{~min}$. The IAE value for the experiment with the MPCRG controller was determined to be 391, while that for the experiment with the PI controller only was 608 . Difference in the IAE values amounts to $35 \%$, which is also supported by the profiles shown in the Fig. 3a.

\section{Conclusions}

This paper discusses the improvements of a widely used and simple control strategy by including another control layer based on optimization. The improvement in the control performance was achieved by predicting the future evolution of the PI controller output together with the distillation column behavior. Considering the future behavior of the inner PI controller, shaped setpoint for the inner PI controller was provided. The filtered setpoint was obtained by solving an optimization problem. The experiments show that overshoots in the process variable decreased for the optimally shaped setpoint.

\section{Acknowledgments}

The authors gratefully acknowledge the contribution of the Scientific Grant Agency of the Slovak Republic under the grant no. 1/0403/15, and the financial support of the Slovak Research and Development Agency under the project APVV-15-0007. M. Klaučo is supported by the internal STU grant scheme for young researchers.

\section{References}

Ahmad A, Wahib A (2007) Application of Model Predictive Control (MPC) Tuning Strategy in Multivariable Control of Distillation Column. Reaktor, 11: 66-70.

Armfield (2010) Continuous Distillation Column, Armfield UOP3CC Instruction Manual. http:// discoverarmfield.com/media/transfer/doc/uop3. pdf.

Åström KJ, Hägglund T (2006) Advanced PID Control. ISA - The Instrumentation, Systems, and Automation Society; Research Triangle Park, NC 27709.

Cutler CR, Ramaker BL (1979) Dynamic Matrix Control. Houston, Texas. AIChE $86^{\text {th }}$ National Meeting.

Drgoňa J, Janeček F, Klaučo M, Kvasnica M (2016) Regionless explicit mpc of a distillation column. In European Control Conference 2016, 1568-1573, Aalborg, Denmark. 
Drgoňa J, Klaučo M, Valo R, Bendžala J, Fikar M (2015) Model identification and predictive control of a laboratory binary distillation column. In Fikar, M. and Kvasnica, M., editors, Proceedings of the $20^{\text {th }}$ International Conference on Process Control, Strbské Pleso, Slovakia. Slovak University of Technology in Bratislava, Slovak Chemical Library.

Gilbert E, Kolmanovsky I (2002) Nonlinear tracking control in the presence of state and control constraints: a generalized reference governor. Automatica, 38(12): 2063-2073.

Klaučo M (2016) Modeling of the Closed-loop System with a Set of PID controllers. Technical Report, Slovak University of Technology in Bratislava, Bratislava. http://www.uiam.sk/assets/publication_info.php?id_ pub $=1770$.

Löfberg J (2004) YALMIP: A Toolbox for Modeling and Optimization in MATLAB. In Proc. of the CACSD Conference, Taipei, Taiwan. Available from http:// users.isy.liu.se/johanl/yalmip/.
Martin PA, Odloak D, Kassab F (2013) Robust model predictive control of a pilot plant distillation column. Control Engineering Practice, 21(3): 231-241.

Mihal' M, Svandová Z, Markoš J (2010) Steady state and dynamic simulation of a hybrid reactive separation process. Chemical Papers, 64(2): 193-202.

Muske KR, Badgwell TA (2002) Disturbance modeling for offset-free linear model predictive control. Journal of Process Control, 12(5): 617-632.

Skogestad S (2007) The dos and donts of distillation column control. Chemical Engineering Research and Design, 85(1): 13-23.

Volk U, Kniese D-W, Hahn R, Haber R, Schmitz U (2005) Optimized multivariable predictive control of an industrial distillation column considering hard and soft constraints. Control Engineering Practice, 13(7): 913-927. 